\title{
Engagement im Ruhestand
}


Martin Kohli/Hans-Jürgen Freter/ Manfred Langehennig/Silke Roth/ Gerhard Simoneit/Stephan Tregel

\section{Engagement im Ruhestand}

Rentner zwischen Erwerb,

Ehrenamt und Hobby

Leske + Budrich, Opladen 1993 
Gedruckt mit Unterstützung der Deutschen Forschungsgemeinschaft

ISBN 978-3-322-93736-0 ISBN 978-3-322-93735-3 (eBook)

DOI 10.1007/978-3-322-93735-3

(C) 1993 by Leske + Budrich, Opladen

Das Werk einschließlich aller seiner Teile ist urheberrechtlich geschützt. Jede Verwertung außerhalb der engen Grenzen des Urheberrechtsgesetzes ist ohne Zustimmung des Verlags unzulässig und strafbar. Das gilt insbesondere für Vervielfältigungen, Übersetzungen, Mikroverfilmungen und die Einspeicherung und Verarbeitung in elektronischen Systemen.

Satz: Leske + Budrich, Opladen 


\section{Vorbemerkung}

Die empirische Untersuchung, die diesem Buch zugrunde liegt, ist im März 1988 mit einem Bericht an die Deutsche Forschungsgemeinschaft abgeschloBen worden. Daß sich die Fertigstellung der Buchfassung so lange verzögert hat, liegt an den Problemen, die aus dem System des akademischen Arbeitsmarktes und der Projektförderung erwachsen. Aufgrund der Notwendigkeit, neue Projekte zu akquirieren und zu bearbeiten, und - für einige Mitarbeiter - aufgrund der Notwendigkeit, andere Stellen anzutreten und sich mit ganzer Energie auf die neuen Aufgaben zu konzentrieren, hat immer wieder die Muße gefehlt, die für eine gründliche Überarbeitung der einzelnen Untersuchungsteile und ihres Zusammenhangs erforderlich war.

Allerdings scheint uns die Verzögerung an der Geltung unserer Überlegungen und Befunde nichts zu ändern. Das Altern der Gesellschaft veraltet nicht. Unsere Untersuchung zielt auf Strukturen und Lebensformen, deren Verbreitung und Bedeutung eher zu- als abnehmen wird. Der publizistische Gefechtslärm, den das Altern der Gesellschaft für kurze Zeit zu entfachen begann (beispielhaft sei die SPIEGEL-Titelgeschichte Kampf der Generationen: Jung gegen alt vom 31.7.1989 genannt), hat sich inzwischen zwar unter dem Ansturm neuer Themen wieder gelegt, aber das Problem selber ist damit nicht verschwunden. Daß die ersten Pulverdämpfe schon verzogen sind, gibt uns vielleicht die Chance zu einer weniger aufgeregten Erörterung der neuen Lebensformen, die mit dem zunehmenden gesellschaftlichen Gewicht des Alters und der Älteren zu entstehen beginnen.

Eine thematische Einschränkung wird mit dem Entstehungszeitraum der Untersuchung schon angezeigt: Sie steht noch ganz im Horizont der alten Bundesrepublik. Von der Umwälzung der Lebensbedingungen durch den Untergang des Realsozialismus und die deutsche Vereinigung ist in ihr noch nichts zu spüren. Aber ihre Aktualität wird durch die Folgen der Umwälzung nicht gemindert, sondern erhöht: Die massive „Freisetzung“ der Älteren aus der Erwerbsarbeit macht die Frage nach alternativen Tätigkeitsformen umso bedeutungsvoller. 
Zwei andere Einschränkungen haben wir dagegen ganz bewußt vorgenommen: Es handelt sich um eine rein qualitative Untersuchung, und sie umfaßt nur Männer. Die Beschränkung auf Männer ist natürlich begründungsbedürftig, umso mehr als Frauen ja im Alter stark in der Mehrheit sind. Die Geschlechterproportion ist in Deutschland aufgrund der Kriegsfolgen besonders unausgeglichen; sie beträgt im alten Bundesgebiet bei den über 60jährigen rund 2:1. Die Beschränkung hat - wie noch zu erläutern sein wird - theoretische und forschungspraktische Gründe. Wir wollen jedenfalls gerade nicht unterstellen, die Männer seien mit den Menschen insgesamt gleichzusetzen. In einer Folgeuntersuchung haben wir unsere Fragestellung auf die Frauen erweitert und entsprechend auch andere Tätigkeitsformen (insbesondere die Pflege von Angehörigen) mit aufgenommen. Zugleich haben wir die qualitativen Fallstudien durch einen Survey ergänzt, der für eine lokale Bevölkerung (nämlich diejenige eines ausgewählten Berliner Stadtbezirks) repräsentativ ist. Die Ergebnisse dieser Folgeuntersuchung werden in Kürze vorliegen, konnten aber in diesem Buch noch nicht berücksichtigt werden.

Eine Anmerkung zum Sprachgebrauch: Obwohl der größte Teil der Felderhebungen jeweils von einem einzigen Mitglied unserer Projektgruppe durchgeführt wurde, haben wir bei der Darstellung der Ergebnisse überwiegend die erste Person Plural beibehalten. Das ist nicht der übliche pluralis majestatis; wir wollen damit festhalten, daß es sich bei der ganzen Untersuchung um eine Gemeinschaftsarbeit handelt. Ohne ständige gegenseitige Unterstützung und Kritik unter allen sechs Autoren wäre sie nicht zustandegekommen. Dennoch sind wir aus offensichtlichen Gründen auch daran interessiert, unsere Beiträge $\mathrm{zu}$ individualisieren. Bei den einzelnen Kapiteln ist verzeichnet, wer von uns dafür die Hauptarbeit geleistet hat und die Hauptverantwortung trägt. Martin Kohli hat das Projekt geleitet, große Teile des Berichts überarbeitet und die Endredaktion übernommen. Hans-Jürgen Freter und Manfred Langehennig haben das Projekt mit konzipiert und seine theoretischen und methodischen Perspektiven stark mitbestimmt. Silke Roth, Gerhard Simoneit und Stephan Tregel brachten die Erfahrungen mit ihren jeweiligen Tätigkeitsgruppen in die gemeinsame Arbeit ein.

Unser erster Dank gilt den Befragten, die uns an ihrer Lebenssituation teilhaben ließen, und den Kooperationspartnern, die uns den Zugang zu den einzelnen Tätigkeitsfeldern ebneten. Wir danken - neben allen, die privat unter dieser Arbeit gelitten haben - unseren Kolleginnen und Kollegen der Arbeitsgruppe Lebenslauf- und Alternsforschung, insbesondere Cornelia Heinz und Inge Brauer, die uns mit großer Einsatzfreude und Fachkunde bei den Verwaltungs- und Korrekturarbeiten unterstützten, und Dietmar Rost, der in gleicher Weise die oft mühseligen Formatierarbeiten leistete, sowie Kai Brauer, der den Ausdruck des Textes besorgte. Wir danken auch der Deutschen Forschungsgemeinschaft für die finanzielle Unterstützung des Projekts, 
den beiden Mitherausgebern der Reihe „Biographie und Gesellschaft“, Werner Fuchs-Heinritz und Fritz Schütze, für ihr Interesse an der Veröffentlichung und unserem Verleger, Edmund Budrich, dessen Geduld wir auf eine harte Probe gestellt haben, für seine unbeirrbar freundliche Ermutigung.

Berlin, im Sommer 1992

M.K. /H.-J.F./M.L./S.R. /G.S./S.T. 


\section{Inhaltsverzeichnis}

Vorbemerkung

1 Fragestellung und theoretische Grundlagen

(Martin Kohli) ............................................ 13

1.1 Das Problem: Altern in der Arbeitsgesellschaft ................ 13

1.2 Aktivität als zentrales Konzept der Sozialgerontologie .......... 19

1.3 Der Ruhestand als Teil der Normalbiographie .................. 25

$1.4 \quad$ Vergesellschaftung durch Arbeit ........................... 31

1.5 Tätigkeitsformen im Vergleich $\ldots \ldots \ldots \ldots \ldots \ldots \ldots \ldots \ldots \ldots \ldots . \ldots \ldots \ldots \ldots$

2 Methodischer Ansatz

(Hans-Jürgen Freter, Martin Kohli, Manfred Langehennig) ..... 45

$2.1 \quad$ Grundoptionen des Designs ................................... 45

2.2 Teilnehmende Beobachtung ............................... 46

2.3 Biographisch-narratives Interview ........................ 51

3 Vergesellschaftung durch Teilzeitarbeit

(Hans-Jürgen Freter) ................................... 59

3.1 Teilzeitarbeit im Ruhestand und ihre Bedingungen ............. 59

3.1.1 Erwerbsbeteiligung im Alter ............................... 59

3.1.2 Die Auswahl der Tätigkeitsgruppe ......................... 67

3.1.3 Rentnerarbeit im Wachbereich ............................. 70

3.1.4 Sozialstrukturelle Bedingungen der Tätigkeit ................. 71

3.2 Datenerhebung und Stichprobe ............................ 78

3.2.1 Zur Datenerhebung ..................................... 78

3.2.2 Die Stichprobe der Teilzeitarbeitnehmer ..................... 79

3.3 Dimensionen der Vergesellschaftung durch Teilzeitarbeit ...... 82

3.3.1 Soziale Interaktionen .................................... 82

3.3.2 Biographische Kontinuität: Das Deutungsmuster „Rentnerjob“ 85 
3.3.3 Identität ............................................... 91

3.3.4 Erfahrungen gesellschaftlicher Veränderungen ............... 93

3.3.5 Zeitliche Strukturierung des Alltags ........................ 94

3.3.6 Biographische Antizipation und Planung .................... 95

3.4 Falldarstellung: Herbert Laubach, Wachmann ................ 96

$4 \quad$ Vergesellschaftung durch selbständige Arbeit

(Stephan Tregel) ............................................ 109

4.1 Selbständige Erwerbsarbeit im Ruhestand und ihre Bedingungen 109

4.1.1 Selbständige im Handwerk .................................... 110

4.1.2 Selbständige im Einzelhandel ............................... 113

4.1.3 Sozialstrukturelle Bedingungen der Tätigkeit ................. 115

4.2 Datenerhebung und Stichprobe ............................ 122

4.2.1 Zur Datenerhebung ......................................... 122

4.2.2 Die Stichprobe der Selbständigen ........................... 123

4.3 Dimensionen der Vergesellschaftung durch selbständige Arbeit 125

4.3.1 Biographische Kontinuität .................................. 125

4.3.2 Identität ................................................ 128

4.3.3 Soziale Interaktionen ..................................... 129

4.3.4 Erfahrungen gesellschaftlicher Veränderungen ................ 130

4.3.5 Zeitliche Strukturierung des Alltags ......................... 131

4.3.6 Biographische Antizipation und Planung .................... 132

$4.4 \quad$ Zwei Falldarstellungen .................................... 133

4.4.1 Fritz Heinkel, Tischler .................................... 133

4.4.2 Hans Grautscho, Lebensmitteleinzelhändler .................. 138

5 Vergesellschaftung durch ehrenamtliche Tätigkeit im sozialen Bereich (Silke Roth und Gerhard Simoneit) .................... 143

5.1 Ehrenamtliche Tätigkeit im sozialen Bereich und ihre

Bedingungen ................................................. 143

5.1.1 Umfang und Relevanz der ehrenamtlichen Tätigkeit im sozialen Bereich ..................................................... 143

5.1.2 Rahmenbedingungen der ehrenamtlichen Tätigkeit in Verbänden der Freien Wohlfahrtspflege ................................. 146

5.1.3 Die Auswahl der Tätigkeitsgruppe .......................... 147

5.1.4 Sozialstrukturelle Bedingungen der Tätigkeit ................. 150

5.2 Datenerhebung und Stichprobe .............................. 153

5.2.1 Zur Datenerhebung ........................................ 153

5.2.2 Die Stichprobe der Ehrenamtlichen im Sanitätswesen .......... 153

5.3 Dimensionen der Vergesellschaftung durch ehrenamtliche Tätigkeit ................................................... 154

5.3.1 Biographische Kontinuität .............................. 154 
5.3.2 Soziale Interaktionen ...................................... 159

5.3.3 Erfahrungen gesellschaftlicher Veränderungen ................. 167

5.3.4 Identität ..................................................... 169

5.3.5 Zeitliche Strukturierung des Alltags ......................... 171

5.3.6 Biographische Antizipation und Planung ..................... 172

5.4 Falldarstellung: Hans Nück, Ausbilder und Sanitäter beim

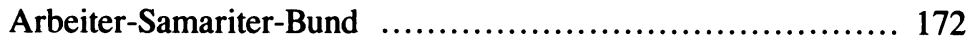

6 Vergesellschaftung durch selbstorganisierte politische Interessenvertretung (Gerhard Simoneit) ...

6.1 Selbstorganisierte politische Interessenvertretung im Ruhestand und ihre Bedingungen

6.1.1 Handlungsfelder politischer Interessenvertretung im Alter ...... 181

6.1.2 Die Auswahl der Tätigkeitsgruppe ........................... 182

6.1.3 Sozialstrukturelle Bedingungen der Tätigkeit ................. 185

6.2 Datenerhebung und Stichprobe ............................ 192

6.2.1 Zur Datenerhebung ......................................... 192

6.2.2 Die Stichprobe der „Seniorenpolitiker“ ..................... 193

6.3 Dimensionen der Vergesellschaftung durch selbstorganisierte politische Interessenvertretung ............................... 194

6.3.1 Biographische Kontinuität ................................ 194

6.3.2 Soziale Interaktionen ...................................... 198

6.3.3 Erfahrungen gesellschaftlicher Veränderungen .................. 199

6.3.4 Identität ....................................................... 199

6.3.5 Zeitliche Strukturierung des Alltags ........................ 202

6.3.6 Biographische Antizipation und Planung .................... 203

6.4 Falldarstellung: Günther Tamm, Seniorenvertreter ............ 204

7 Vergesellschaftung im Hobby-Bereich (Manfred Langehennig) ..................................... 213

7.1 Hobby-Tätigkeit und ihre Bedingungen .......................... 213

7.1.1 Organisierte Hobby-Aktivitäten und die Mitgliedschaft in Hobby-Vereinen ............................................. 213

7.1.2 Die Untersuchungsfelder und die Auswahl der Tätigkeitsgruppen 214

7.2 Datenerhebung und Stichprobe .............................. 220

7.2.1 Zur Datenerhebung ........................................ 220

7.2.2 Die Stichprobe der Hobbyisten ............................ 222

7.3 Dimensionen der Vergesellschaftung durch Hobby-Tätigkeit .... 223

7.3.1 Soziale Interaktionen ......................................... 223

7.3.2 Erfahrungen gesellschaftlicher Veränderungen ................ 244

7.3.3 Zeitliche Strukturierung des Alltags ......................... 256

7.3.4 Biographische Kontinuität ................................. 260 
7.3.5 Biographische Antizipation und Planung .................... 264

7.3.6 Identität ................................................ 267

7.4 Falldarstellung: Gustav Must, Ansichtskartensammler ......... 268

8 Engagement im Ruhestand: Ein zusammenfassender

Vergleich (Hans-Jürgen Freter und Martin Kohli) ............... 275

8.1 Bedingungen des Zugangs .............................. 275

8.2 Dimensionen der Vergesellschaftung ....................... 280

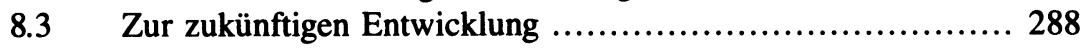

Literatur ................................................... 293 